\title{
DIABETIC RETINOPATHY IMAGE CLASSIFICATION USING DEEP NEURAL NETWORK
}

\author{
PARVATHY EN, BHARADWAJA KUMAR G
}

School of Computing Science and Engineering, Vellore Institute of Technology, Chennai, Tamil Nadu, India. E-mail: asha.s@vit.ac.in

Received: 19 January 2017, Revised and Accepted: 20 February 2017

\begin{abstract}
Healthcare is an important field where image classification has an excellent value. An alarming healthcare problem recognized by the WHO that the world suffers is diabetic retinopathy (DR). DR is a global epidemic which leads to the vision loss. Diagnosing the disease using fundus images is a timeconsuming task and needs experience clinicians to detect the small changes. Here, we are proposing an approach to diagnose the DR and its severity levels from fundus images using convolutional neural network algorithm (CNN). Using CNN, we are developing a training model which identifies the features through iterations. Later, this training model will classify the retina images of patients according to the severity levels. In healthcare field, efficiency and accuracy is important, so using deep learning algorithms for image classification can address these problems efficiently.
\end{abstract}

Keywords: Diabetic retinopathy, Convolutional neural network, Fundus photography.

(C) 2017 The Authors. Published by Innovare Academic Sciences Pvt Ltd. This is an open access article under the CC BY license (http://creativecommons. org/licenses/by/4. 0/) DOI: http://dx.doi.org/10.22159/ajpcr.2017.v10s1.20512

\section{INTRODUCTION}

Artificial neural network (ANN) is inspired from the brain. The brain is considered as the information processing device of a human. The brain has many abilities and can excel many engineering products in fields such as vision, speech recognition, and learning. The ANN model mimics the properties of the brain which are neurons and its property of working parallelly. The neurons in the brain are connected to each other as synapses which help in parallel computation. Convolutional neural networks (CNNs) are an important class of neural networks that have been used to solve numerous computer vision problems. Deep CNNs, in particular, are composed of several layers of processing called convolutional layers and pooling layers that allow the neural network to snare prolific images properties [1]. In the present work, we would like to propose a methodology using CNNs for classifying the images that indicate diabetic retinopathy (DR).

The rampancy of diabetes among all age groups worldwide was estimated to be $2.8 \%$ in 2000 and $4.4 \%$ in 2030 . The increase in number of people with diabetes is protruded to rise from 171 million in 2000 to 366 million in 2030 [2]. Diabetic causes a variety of eye diseases [3] that can be described as follows: (a) DR affects blood vessels in the retina that leads to vision loss among people with diabetes and the dominant cause of vision impairment and blindness, (b) diabetic macular edema is a repercussion of DR and causes swelling in an area of the retina called the macula, (c) cataract is a clouding of the eye's lens which is 2-5 times more likely to develop in patient with diabetes than those without diabetes. Cataract also tends to develop at an earlier age in people with diabetes, and (d) glaucoma is a decease that damages the eye's optic nerve and is having doubled risk in case of patients with diabetes. Hence, all the different forms of diabetic eye disease can cause severe vision loss and blindness. According to the WHO Global Report on diabetes, in 2014, approximately 422 million people are suffering from diabetes, whereas in 1980, it was 108 million [4]. Diabetes is becoming a global epidemic. Every patient with diabetes is at the risk of developing DR. Surveys on DR say that $35 \%$ of patients with diabetes have some degrees of DR. DR is a disease which frequently leads to the poor visual functioning, and in working-age populations, it is the leading cause of blindness. The tragedy of this disease is when we find that those blinded by diabetes are quite young, in their twenties or thirties. Moreover, DR does not have any cure if loss of vision occurs only treatment is to sustain the current vision.
DR as said is a global epidemic which leads to vision loss. In DR, the blood vessels in retina will go through series of changes which can be explained as severity levels for DR. There are four severity levels for DR (Fig. 2), the first severity level is mild non-proliferative retinopathy. In severity level one, microaneurysms will form, it is

a balloon-like swelling in the blood vessels of the retina. In moderate non-proliferative retinopathy, the second severity level, the blood vessels will break and leakage occurs. Severe non-proliferative retinopathy is the third severity level where the blood vessels are blocked vessels and prevent blood flow to some parts of the retina and it also triggers to form new retinal blood vessels. Proliferative DR is the fourth level new blood vessels which are weaker than the normal blood vessels will form. These fragile blood vessels grow along the inside surface of the retina will make more leakage. In this stage, retina will be pulling away from underlying tissue which causes permanent vision loss.

The retina images are from fundus photography. This photography uses fundus camera to get the color images of interior surface of the eye so that we can monitor the eye and find the disorders. Fundus camera contains intricate microscope which attached to a flash-enabled camera. This camera helps to photograph the interior surface of the eye including retina, macula, and posterior pole. Currently, the DR detection is a manual and time-consuming process. The patient will go to clinic to take fundus photograph of the retina and the image will take. He/ she will get the result in 2 or 3 days, and then patient should take the result to an ophthalmologist for the review. As this traditional way of DR detection takes more time and the chance of miscommunication and the delayed treatment lead is more, there we can use automatic DR detection algorithm which can be implemented in fundus camera itself. Here, after taking the retina image, the image will be given to already trained model which classifies the images based on whether the patient has DR or not, if he/she has the DR, and then the severity level of the disease. This helps both the ophthalmologist and patient so that the delayed treatment would not be happen.

\section{RELATED WORK}

Niemeijer et al. [5] proposed an algorithm that is capable of detecting exudates, cotton-wool spots, and drusen and differentiating among them on color images of the retina. The area under the ROC curve of 0.95 shows that the system has sensitivity and specificity almost equivalent to that of human retinal experts. Casanova et al. [6] proposed random forests based methods to DR classification analyses based 
on fundus photography data. Verma et al. [7] proposed a method for classification of the different stages of eye disease based on the area and perimeter of the blood vessels and hemorrhages using random forests technique. Ahmad et al. [8] reviewed the latest techniques in digital image processing and pattern classification employed for the detection of DR and compared them. Ravishankar et al. [9] proposed a robust approach for the localization of the different features and lesions in a fundus retinal image and showed that many of the features such as the blood vessels, exudates and microaneurysms, and hemorrhages can be identified a quite scrupulous using different morphological-based operations applied in appropriate manner.

\section{ARCHITECTURE AND METHODOLOGY}

\section{CNNs}

Image classification can be done by supervised and unsupervised. In supervised learning, a training model will be made by analyzing the features of training data. Based on this training model, the new data will be classified. CNN is an algorithm for classification in supervised learning method. CNN is a type of feed-forward ANN which inspired by the neurons and brain. It is a variation of multilayer perceptron which needs only less pre-processing techniques and it is easier to train. CNN is comprised convolutional layers, pooling layers, and fully connected layers. Fig. 3 shows the three layers of CNN.

\section{Convolutional layers}

The core building block of CNN is convolutional layer. The layer contains a set of learnable filters and these filters have small receptive fields. In each forward pass, it will produce a two-dimensional map of corresponding filter by computing the dot product between input and entries of filter. By this, the network will learn about the filters which are activated when specific feature at specific spatial position occurs. The depth dimension with activation maps of each filter will form the full output volume of convolutional layer.

\section{Pooling layers}

Pooling is a non-linear down sampling. To implement the pooling layer, the most common non-linear function. The max pooling layer partitions the input image into congregation of non-overlapping rectangles and the layer will output the maximum for each subregion. If a feature is identified, then this helps in finding the rough location relative to other features. The intermediate representation's dimensionality will be reduced by max pooling. Pooling layers are inserted in between convolutional layers.

\section{Fully connected layers}

The fully connected layers do the high-level reasoning of a neural network. This layer connects all the neurons of the previous layers (convolutional or pooling or connected). The activations can be computed by a matrix multiplication, and then a bias offset will be added.

Image classification using CNN is used for the task of classification of DR images in this work. Fundus retina images are used as the dataset. As we are using supervised learning, we need both training and test dataset. In training phase, we need to do some pre-processing steps. Fig. 4 shows the workflow of the present work.

\section{Preprocessing}

As we are using CNN algorithm, only a little preprocessing is needed. Here, only resizing the images and rotation is sufficient. Rotating image in $45^{\circ} \mathrm{C}, 90^{\circ} \mathrm{C}$, and $180^{\circ} \mathrm{C}$ should be done. These rotations will add more images to the training dataset which helps to get an efficient training model. After pre-processing step, the pre-processed dataset will be given to the CNN algorithm.

\section{Training phase}

In training phase, the input of the CNN algorithm will be the preprocessed training data. As the input goes iteratively through each

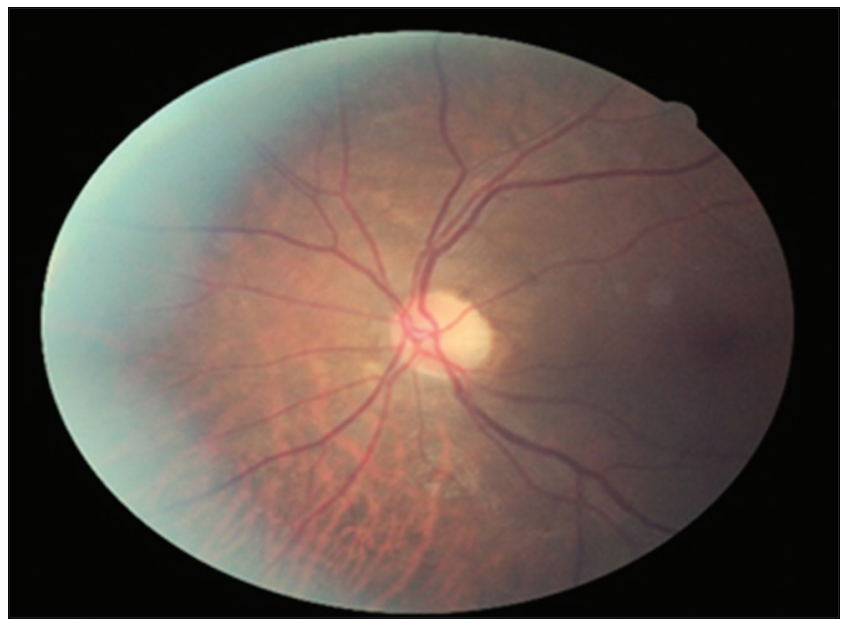

Fig. 1: Normal retina

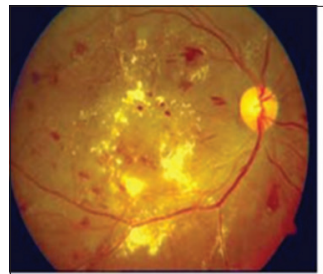

Severity 1

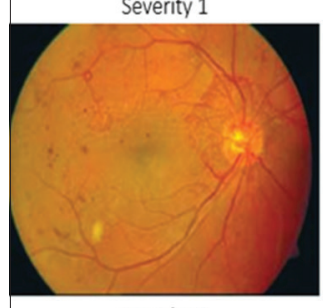

Severity 3

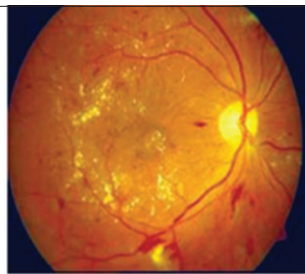

Severity 2

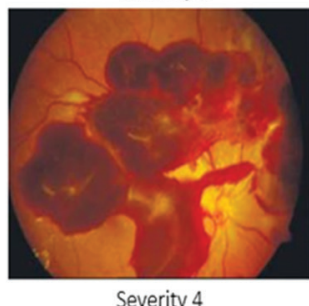

Severity 4

Fig. 2: Severity levels

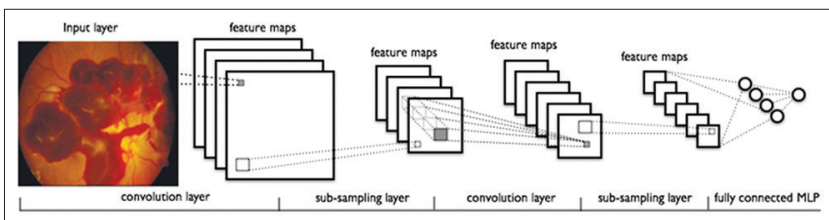

Fig. 3: Architecture of convolutional neural network

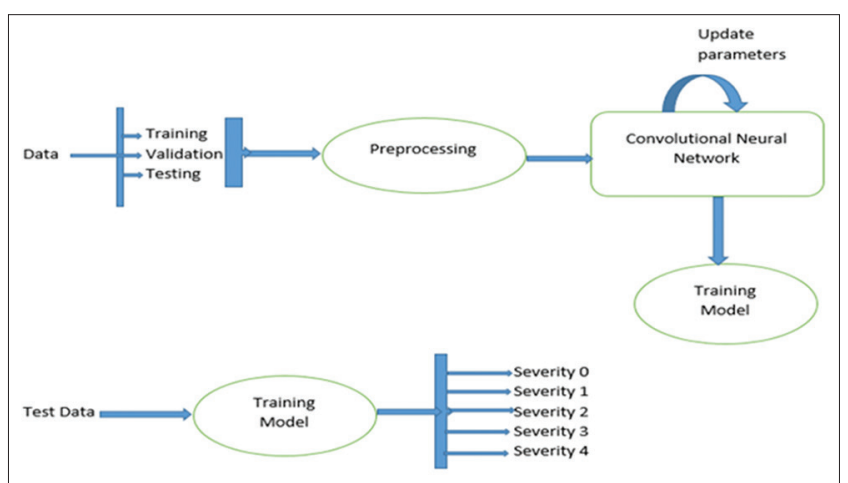

Fig. 4: Workflow

layer (convolutional, pooling, and fully connected), it will identify the features in the images. The layers in the algorithm will find the best 
features which are needed for classification using feature maps. There must be at least six layers in the algorithm to get a model. When the number of layers are increasing, the training model will be more and more efficient. About nine convolutional layers, eight max pooling layers and two fully connected layers are the combination of layers we would like to use in the present work. This combination will make the algorithm to work more accurately and efficiently. We can easily classify the image with these number of layers.

\section{Convolution layer}

Consider we are having $\mathrm{N}^{*} \mathrm{~N}$ squared neuron layer and it is followed by convolution layer. The filter which we are using is $\mathrm{m}^{*} \mathrm{~m}$ one which is $\omega$. Then the output of the convolutional layer will be of the size $(\mathrm{N}-\mathrm{m}+1)^{*}(\mathrm{~N}-\mathrm{m}+1)$. To get the pre non-linearity input, we have to sum up the output from the previous layer which is represented by $\mathrm{x}_{\mathrm{ij}}^{1}$.

$$
x_{i j}^{l}=\sum_{a=0}^{m-1} \sum_{b=0}^{m-1} \omega_{a b} y_{(i+a)(j+b)}^{l-1}
$$

Then, the nonlinearity of the convolution layer is

$$
y_{i j}^{i}=\sigma\left(x_{i j}^{i}\right)
$$

In addition to sum up the weights, we have to propagate errors back to the previous layers. We use chain rule for finding the errors and propagating it back.

\section{Max pooling layer}

This is a simple layer. It will select a $\mathrm{k}^{*} \mathrm{k}$ region and the output of the region will be maximum value in that particular region. If $\mathrm{N}^{*} \mathrm{~N}$ is the input layer, then the output will be $(\mathrm{N} / \mathrm{k})^{*}(\mathrm{~N} / \mathrm{k})$. We will get this output by reducing each single $\mathrm{k}^{*} \mathrm{k}$ region by maxfunction. The reduction happens in the forward propagation and the error from the previous layer is back propagated.

\section{Testing phase}

During testing, the model will find the features of images and classifies according to it. In DR detection, the training model has 5 classes. The first one is severity 0 which state that patient does not have the disease. The other classes are severity 1 , severity 2 , severity 3 , and severity 4. Severity 4 is the most severe condition of the disease where the patient loses his/her vision. The algorithm will first classify whether the patient is suffering from disease or not. If the patient is suffering from the disease, then it will check the severity level of his disease. The output for this will be the patient's severity level. Now, from this output, the doctor can take quick action based on the severity levels. Like this, the doctor can prevent the vision loss happening in the patient.

\section{CONCLUSION AND FUTURE WORK}

CNN is the deep learning algorithm which will work efficiently and accurately for image classification. In this paper, a method using CNN algorithm is proposed to detect the DR and classifying the images per severity level of the disease. As the treatment is important in DR, this approach will reduce the time consumption and efficiently diagnose the disease so that there would not be any delay in the treatment. The algorithm will be implemented in the system which is connected to fundus camera. Whenever the camera takes an image, the system will pass it to the algorithm where the algorithm detects whether the corresponding patient has DR or not, and if it is detected, then the severity level of the disease.

\section{REFERENCES}

1. Liu B, Liu Y, Zhou K. Image Classification for Dogs and Cats. TechReport, University of Alberta; 2013.

2. Wild S, Roglic G, Green A, Sicree R, King H. Global prevalence of diabetes: Estimates for the year 2000 and projections for 2030. Diabetes Care 2004;27(5):1047-53.

3. Available from: https://www.nei.nih.gov/health/diabetic/retinopathy.

4. World Health Organization. Global Report on Diabetes; 2016.

5. Niemeijer M, van Ginneken B, Russell SR, Suttorp-Schulten MS, Abramoff MD. Automated detection and differentiation of drusen, exudates, and cotton-wool spots in digital color fundus photographs for diabetic retinopathy diagnosis. Invest Ophthalmol Vis Sci 2007;48(5):2260-7.

6. Casanova R, Saldana S, Chew EY, Danis RP, Greven CM, Ambrosius WT. Application of random forests methods to diabetic retinopathy classification analyses. PLoS One 2014;8:e77949.

7. Verma K, Deep P, Ramakrishnan AG. Detection and classification of diabetic retinopathy using retinal images. In: 2011 Annual IEEE India Conference. IEEE; 2011.

8. Ahmad A, Mansoor AB, Mumtaz R, Khan M, Mirza SH. Image processing and classification in diabetic retinopathy: A review. In: Visual Information Processing (EUVIP), $5^{\text {th }}$ European Workshop on. IEEE; 2014.

9. Ravishankar S, Jain A, Mittal A. Automated feature extraction for early detection of diabetic retinopathy in fundus images. In: Computer Vision and Pattern Recognition, CVPR-2009. IEEE Conference on. IEEE; 2009. 